\title{
Response dynamics of stochastic integrate-and-fire neurons
}

\author{
Joanna Pressley ${ }^{1}$, Todd W Troyer ${ }^{2^{*}}$ \\ From Nineteenth Annual Computational Neuroscience Meeting: CNS*2010 \\ San Antonio, TX, USA. 24-30 July 2010
}

The leaky integrate-and-fire (LIF) is the simplest neuron model that captures the essential properties of neuronal signaling while seemingly remaining simple enough to analyze. Yet, a comprehensive description of LIF response to time-varying stochastic input is lacking. As a first step, we survey the linear component of LIF responses over a wide range of baseline input parameters. Using a diffusion approximation, we calculate the relative gain as a function of frequency for three types of sinusoidal input modulations: modulations of the mean input current, modulations of the variance of the input current, and co-modulation of the mean and variance to simulate rate modulation of a Poisson input spike train. The output parameter is the time-dependent probability of producing a spike, i.e. the ensemble firing rate. In accord with common intuitions, we find that the stochastic LIF model displays low pass behavior for modulations of the mean, with the gain going to zero at high frequency. For baseline levels of mean current that are supra-threshold, the gain is enhanced by resonances at modulation frequencies near the baseline firing rate [1]. A quantitative analysis of the low-pass corner frequency shows that when operating at low rates the model responds at higher frequencies than the passive membrane filtering would suggest. Furthermore, at higher baseline frequencies $\mathrm{f}$, cutoff frequencies are in the range of $4 \mathrm{f}$, much greater than the $\mathrm{f} / 2$ cutoff expected from Nyquist arguments. For modulations in the input variance, we also find peaks in the gain function but these peaks persist into the regime of fluctuation-driven firing and the peak of these resonances does not correspond to the baseline firing rate. In accord with previous studies [2], gain values approach one at high frequencies across parameters. However, the low frequency gain is highly regime-dependent, with the low frequency gain much greater than one in the fluctuation-driven regime. The low-frequency gain is also highly regime-dependent in response to modulations in Poisson input rate. However, for frequencies greater than the inverse of the membrane time constant, the gain curves, for all baseline parameters, coalesce into a common trajectory toward a gain of one. Several of these response properties can be understood by noting that the ensemble firing rate depends on a multiplicative interaction between separate underlying factors, one of which is proportional to the present value of the input variance and the other which depends on the dynamics of integration and reset. However, further work will be needed to understand the response at frequencies significantly greater than expected from simple membrane filtering or firing rate sampling, and to understand the nature of the firing rate resonances in response to modulations of input variance.

\section{Author details}

'Department of Mathematics, Vanderbilt University, Nashville, Tennessee 37240, USA. '2Department of Biology, University of Texas, San Antonio, Texas, 78249, USA.

Published: 20 July 2010

\section{References}

1. Knight BW: The relationship between the ring rate of a single neuron and the level of activity in a population of neurons. experimental evidence for resonant enhancement in the population response. $J$ Gen Physiology 1972, 59(6):767-778.

2. Lindner B, Schimansky-Geier L: Transmission of noise coded versus additive signals through a neuronal ensemble. Phys Rev Lett 2001, 86(14):2934-2937.

\section{doi:10.1186/1471-2202-11-S1-P22}

Cite this article as: Pressley and Troyer: Response dynamics of stochastic integrate-and-fire neurons. BMC Neuroscience 2010 11(Suppl 1):P22.

* Correspondence: todd.troyer@utsa.edu

${ }^{2}$ Department of Biology, University of Texas, San Antonio, Texas, 78249, USA 